\title{
Performance Evaluation of a Compression Algorithm for Wireless Sensor Networks in Monitoring Applications
}

\author{
Ivanovitch M. D. Silva \\ Luiz Affonso Guedes \\ Department of Engeneering Computer and Automation \\ Federal University of Rio Grande do Norte \\ Campus de Lagoa Nova, Natal, RN, Brazil \\ \{ivan,affonso\}@dca.ufrn.br \\ Francisco Vasques \\ Department of Mechanical Engineering \\ University of Porto \\ Porto - Portugal \\ vasques@fe.up.pt
}

\begin{abstract}
Wireless sensor network (WSN) is an emerging technology that targets multiple applications in the different environments. Its infrastructure is composed of a large number of sensor nodes with a limited physical capacity and low cost. The energy consumption must be as optimized as possible in order to extend its lifetime. The use of data compression techniques can be an advantage in the WSN context, once these techniques eliminate transmission of redundant information and consequently can be adopted to minimize the consumption of energy in the sensor nodes. WSN for monitoring applications can benefit from this technique as it may maximize the lifetime of batteries.

The main motivation of this paper is to investigate the performance of a data compression algorithm for WSN in the context of monitoring applications. To validate the proposal, simulation experiments have been performed using the Network Simulator (NS-2) tool.
\end{abstract}

\section{Introduction}

Wireless sensor network (WSN) is an emerging technology that is being applied to ensure connectivity in the different environments. This property enables WSN to extract information in a way simpler than the traditional monitoring and instrumentation techniques [8]. However, a series of new challenges must still be overcome, such as the improvements of information security, fault tolerance and quality of service (QoS) properties.

Structured networks are composed by a large number of devices, named as sensor nodes. These nodes are char- acterized by a limited amount of sensing, processing and communication capabilities, and have also limitations due to energy supply and very small physical size. Sensor nodes work on a cooperative manner in order to disseminate information through the network. Sink nodes get information from network and make it available for client applications. The installation of sensor nodes can be done either without a previously defined structure [5], or with a structured one [11], depending on the supported application that will be installed.

Generally, as the sensor nodes are distributed over large areas of difficult access, they must operate without human intervention for a long period of time [2]. Therefore the energy consumption must kept as low as possible, otherwise the nodes may exhaust their operating energy and will be turn-off. The frequent replacement of sensor nodes can reduce significantly the benefits brought by wireless sensor networks and in general must be avoided. In short, the energy consumption in the sensor nodes must be as optimized as possible, avoiding the decrease of network performance in the event of several consecutive disconnections of sensor nodes.

As WSN have many devices, it is possible to have multiple elements monitoring the same variable set in the environment, causing duplication of information on the network. This feature provides a level of redundance for WSN, as the effect of message losses can be minimized. On the other hand, it is important to say that the transmission process is the activity that consumes more energy in the sensor nodes. Therefore, solutions for optimizing the routing and data transmission must be implemented in order to minimize the energy consumption. In this context, data compression algorithm can be used as a technique to minimize the energy consumption during the transmission 
process, whether by reduction of message size or by elimination of the redundant data transmission.

In this paper we are interested about the monitoring applications for industrial automation environments, where the size of transmitted messages is tipically very small [13]. Therefore, we belive that the adoption of a compression algorithm that minimize the message sizes have a worse performance than a temporal compression algorithm for the minimization of energy consumption. Thus, we will focus this work only on a temporal data compression approach.

The Swinging Door [4] is a very popular algorithm for temporal data compression. It is based on a temporal structure with low complexity of implementation, which aims to eliminate redundant data transmission. Wireless sensor network for monitoring applications can benefit from the use of this technique to maximize the batteries lifetime in the various sensor nodes, thus extending the required maintenance time for the system as a whole.

Therefore, in this paper we investigate the performance of a WSN when the sensor nodes have been equipped with the Swinging Door data compression algorithm. The target of this investigation is to assess the benefit that can be brought by such kind of algorithm, in what concerns the lifetime of nodes in a WSN. The proposal is validated by simulate results using NS2 Simulator in monitoring applications.

The paper is organized as follows. In Section 2, we give a brief description about wireless sensor network and its most relevant protocols. Next, in Section 3, we outline the Swinging Door algorithm, that was chosen as the temporal data compression technique addressed in this paper. The Section 4 shows the simulation models and the obtained results. Finally, Section 5 concludes the paper and indicates directions for future works.

\section{Wireless Sensor Network}

Wireless sensor network is a pervasive technology targeting the connectivity between sensor nodes in multiple environments. Its infrastructure may be composed of a large number of sensor nodes, which have relative inexpensive computational process and very small physical size. The sensor nodes in monitoring applications measure local environmental conditions and forward the sensed values to a set of central points, named sink nodes, for appropriate processing. Sensor nodes can sense the environment, communicate with their neighboring nodes, and perform basic computations on the collected data [9]. Installation flexibility and configuration for sensor nodes enable a better usability and maintenance than traditional communication technologies [6]. These characteristics allow the used of WSN in a wide range of useful applications.

Currently, WSNs use solutions based either on standardized or proprietary protocols. There are many different protocols related with the upper layers, but the IEEE
802.15.4 protocol [1] is a de facto standard for the lower layers. The IEEE 802.15.4 protocol defines the characteristics of the physical and medium access control for low-rate wireless personal area network (LR-WPAN). In folowing, we will present some relevant aspects about the physical and data-link layers of the IEEE 802.15.4.

\subsection{IEEE 802.15.4 - Physical Layer}

The physical layer can operate in 3 distinct frequency bands: $2450 \mathrm{MHz}$ ISM band (worldwide) with 16 channels, $915 \mathrm{MHz}$ band (only in the US) with 10 channels and $868 \mathrm{MHz}$ band with only 1 channel (European and Japanese standards). All the support bands use the Direct Sequence Spread Spectrum (DSSS) access mode. Signaling in the $2450 \mathrm{MHz}$ band is based on Orthogonal Quadrature Phase Shift Keying (O-QPSK) while the $868 / 915 \mathrm{MHz}$ bands rely on Binary Phase Shift Keying (BPSK). The throughputs of bands are $250 \mathrm{kbps}, 40 \mathrm{kbps}$ and $20 \mathrm{kbps}$, respectively. Besides, the physical layer management services include the activation and desactivation of the radio transceiver, energy detection (ED) within the current channel, link quality indication (LQI) for received packets, clear channel assessment (CCA) for carrier sense multiple access with collision avoidance (CSMA-CA), channel frequency selection and data transmission and reception.

\subsection{IEEE 802.15.4 - Data-link Layer}

The Medium Access Control (MAC) is part of the Data-link Layer. It defines two types of devices: Reduced Function Device (RFD) and Full Function Device (FFD). RFDs are equipped with a limited set of functions. They can act as end-devices and their communications are restrict for FFDs. On the other hand, FFDs are equipped with full set of functions and can act as a PAN coordinator or an end-device. In this case, there are not any communication restrictions.

The IEEE 802.15.4 standard defines operation modes based or not on a superframe structure. In the first case, a superframe structure is used to synchronize the network communication. The standard defines one PAN information base (PIB), named as macBeaconOrder (BO), to activate this operation mode. $\mathrm{BO}$ assumes values from 0 up to 14 . The figure 1 shows the superframe structure.

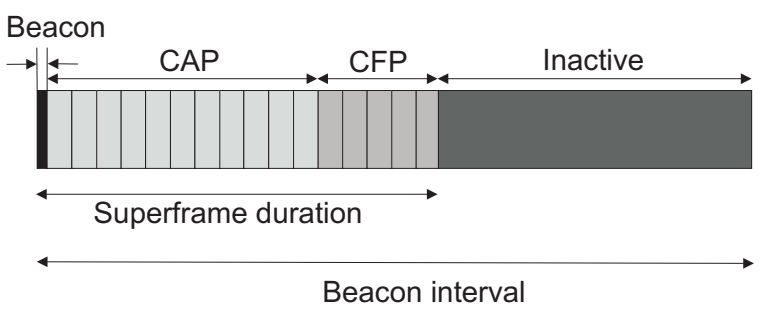

Figure 1. Superframe structure.

The superframe structure is divided into active (CAP and CFP) and inactive portions. A beacon slot delimits the 
start of the superframe. The active portion is divided in 16 equally sized slots. The sensor nodes compete for channel access using a slotted CSMA-CA protocol during the contention access period (CAP). However, applications with low-latency requirements must use the contention free period (CFP) in order to access the medium without competing for it. During the inactive portion, the sensor nodes may enter in a low-power mode to save energy.

The superframe duration (active portion) and beacon interval (active portion + inactive portion) are defined by two PIB: SO (macSuperframeOrder) and BO. These variables satisfy the following relation: $0 \leq S O \leq B O \leq 14$. The values of BO and SO are determined by the coordinator. This information will be relevant to configure the simulation tool used in this work.

In this transmission mode, it is possible to transmite from the end-device to the coordinator or vice-versa. The end-device must wait for a beacon message in order to start a communication. After it has received a beacon message, the end-device is enabled to transmit. Then, it transmits the data frame to the coordinator using the slotted CSMA-CA mode. Finally, the coordinator sends an acknowledgment frame to the end-device. This mechanism is described in figure 2 (a).

On the other hand, when the coordinator has to transfer data to a end-device, it uses the beacon message to announce pending messages. To save energy, end-devices sleep in the most of the time and wake up periodically to listen the beacon messages. When a beacon message with a pending information is sensed by an end-device, this has to send a MAC request command to the coordinator indicating that pending information can be transmitted. Then, the coordinator confirms this indication by transmiting an acknowledgment frame followed by the pending data using the slotted CSMA-CA mode. Finally, the end-device sends an acknowledgment frame to the coordinator. This mechanism is described in figure 2 (b).

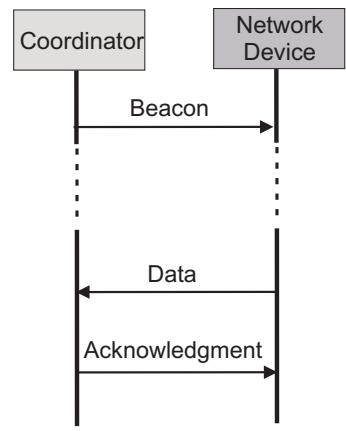

(a)

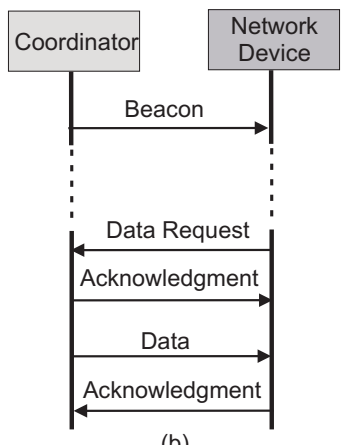

(b)
Figure 2. (a) Communication to a coordinator in a beacon-enabled PAN. (b) Communication from a coordinator in a beaconenabled PAN.

When operating without a superframe structure, there are no beacon frame and the communication happens on the basis of unslotted CSMA-CA. The coordinator is always turned on and the communication from end-devices to the coordinator is poll-based. This operation mode is actived when the $\mathrm{BO}$ value is set to 15 .

Depending on the application requirements, two main types of network topology are supported: the star topology and peer-to-peer topology. In the star topology, enddevices and sink-node (PAN coordinator) send messages to each other. Thus, all the communications involve the sink-node.

Otherwise, in the peer-to-peer topology all types of communication are possible among end-devices, routedevices and sink-nodes. Thus, peer-to-peer topology allows complex network formations to be implemented through a multihop network.

\section{Compression Algorithm Architecture}

Despite the recent advances on routing, data management, security, robustness and fault tolerance in wireless sensor networks, the energy efficiency consumption still is the main focus of development in this area. This trend is motivated mainly by the reduced physical capacity of sensor nodes.

In a WSN, the operation that consumes more energy is just data transmission. Experiments [3] performed in dense scenarios have shown that the data transmission consumes around $50 \%$ of the system's energy. Therefore, solutions for optimizing data transmission must be implemented to minimize the energy consumption. A possible solution to save energy is to use a temporal compression algorithm that eliminates the transmission of redundant information. In this paper we will assess the use of the temporal compression algorithm Swinging Door [4], in a scenario where the target for the use of this algorithm is the minimization of the energy consumption in sensor nodes.

The Swinging Door algorithm is based on concept of timed windows. Basically, the algorithm can optimize the energy consumption because it enables only relevant data to be sent onto the network. Deviation compression, maximum and minimum compression time are the parameters used by the algorithm to create the timed windows, where the relevant data are those outside of the timed window. Thus, no relevant data is transmitted, i.e., they are lost. To recover the original information, interpolation methods can be used.

There are two situation to determine if a current datum is relevant (see algorithm 1): i) when the timeline is greater than or equal to the maximum compression time or ii) when it is the first datum of the new timed window. On the other hand, the previous datum is transmitted when the compression deviation of current datum is outside the timed window and the timeline is not less than 
input : datum- information that will be whether or not transmitted in the specific time, timeline- computation time, tmax, tmin- time boundaries, bias- compression deviation.

output: Confirmation to transmission

if isfirstData (datum) then

currentBaseTime $\leftarrow$ timeline;

currentBase $\leftarrow$ datum;

end

return true;

else if timeline $\geq$ tmax + currentBaseTime then

currentBaseTime $\leftarrow$ timeline;

currentBase $\leftarrow$ datum;

end

return true;

else if abs (currentBase - datum) $\geq$

currentBase $\times$ bias then

if timeline $\geq$ tmin + currentBaseTime then

currentBaseTime $\leftarrow$ timeline;

currentBase $\leftarrow$ dataPrevious;

end

return true;

end

return false

Algoritmo 1: Swinging Door algorithm.

the minimum compression time. The figure 3 shows a data compression example using the Swinging Door algorithm. The compression rate was $50 \%$, where 2 current samples ( 0 and 5 ) and one previous sample (3) were transmitted. It

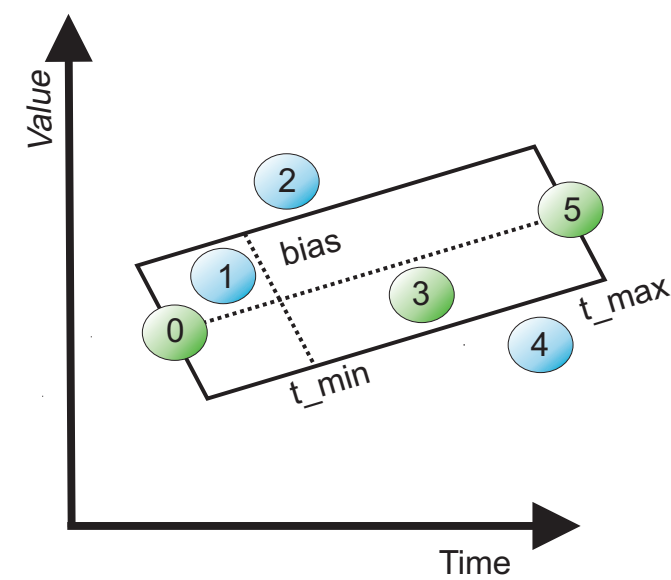

Transmitted data

\section{Compressed data}

Figure 3. Swinging Door example.

is easy to observe that this algorithm has a low-complexity processing. Thus, the adoption of this algorithm is feasible for implementation into wireless sensor nodes, once these devices have a limited physical processing capacity.

In a previous paper [12], using experimental scenarios we have shown that the incorporation of the Swinging Door into a smart sensor can decrease considerably the data exchanges on the communication network. However, an assessment of the overall saved energy by the use of this algorithm was not realized.

\section{Simulation and Results Analysis}

In this paper, we assess the performance of temporal compression algorithm Swinging Door embedded into wireless sensor nodes, in what concerns the overall energy consumption. More specificaly, the NS-2 [7] simulation tool was used to build adequate models for modelling tipical monitoring applications.

The simulation tool provides mechanisms to select the routing protocols, create topologies and applications, configure PAN coordinator, end-devices and router-devices, radio-propagation and antenna model, traffic and queue patterns, link and node model failures, superframe structure and event animation.

The configuration of the NS-2 simulator for our experiments was based on hardware, superframe, energy consumption, topology and routing parameters, as represented in table 1 .

Table 1. Used parameters to simulation.

\begin{tabular}{|c|c|}
\hline \multicolumn{2}{|c|}{ Hardware Parameters } \\
\hline Channel type & Channel/WirelessChannel \\
\hline $\begin{array}{l}\text { Propagation model } \\
\end{array}$ & Propagation/TwoRayGround \\
\hline Queue protocol & Queue/DropTail/PriQueue \\
\hline Queue length & 50 \\
\hline Physical layer & Phy/WirelessPhy/802_15_4 \\
\hline MAC layer & Phy/WirelessPhy/802_15_4 \\
\hline CSThresh & $3.9062 \mathrm{e}-14$ \\
\hline RXThresh & $3.9062 \mathrm{e}-14$ \\
\hline Frequency & $2.4 \mathrm{GHz}$ \\
\hline \multicolumn{2}{|c|}{ Superframe Parameters } \\
\hline BO & 4 \\
\hline SO & 4 \\
\hline \multicolumn{2}{|c|}{ Energy Consumption Parameters } \\
\hline Power transmission & $61.92 \mathrm{~mW}$ \\
\hline Power receive & $44.40 \mathrm{~mW}$ \\
\hline Power idle & $2.40 \mu \mathrm{W}$ \\
\hline Power sleep & $1.44 n \mathrm{~W}$ \\
\hline \multicolumn{2}{|c|}{ Topology and Routing Parameters } \\
\hline Routing protocol & AODV \\
\hline Area & $300 \mathrm{~m} \times 300 \mathrm{~m}$ \\
\hline Range & $50 \mathrm{~m}$ \\
\hline Topology & Star \\
\hline
\end{tabular}

The hardware parameters were configured specifically for a wireless sensor network, where received threshold (RXThresh) and carrier sense threshold (CSThresh) were used to give the sensing range of the nodes. With respect to superframe parameters, SO (macSuperframeOrder) and 
BO (macBeaconOrder) were choosen with values that maximize the percentage of successfuly transmitted data packets [10]. Following, the energy consumption parameters were based on a real Texas Instruments $\mathrm{CC} 2520$ radio transceiver with a very low power consumption.

A star topology has been used in our simulation assessments. It is composed by one PAN coordinator (FFD) and 7 end-devices (RFD), as shown in figure 4. The PAN coordinator is turned on when the simulation is started, but the other devices are turned on only 5 seconds later with delay intervals of 1 second between them. For this configuration, the average network startup time was around 13 seconds.

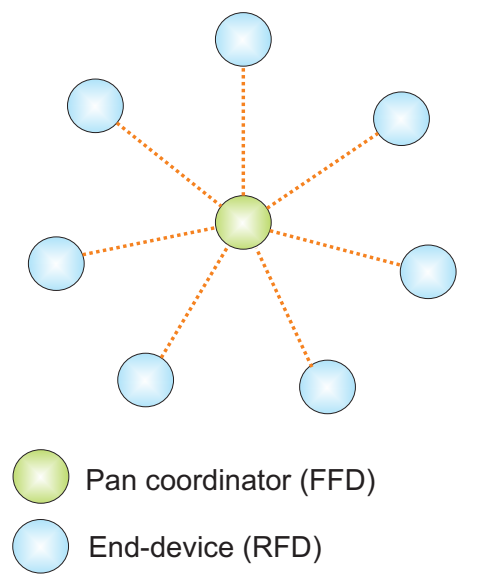

Figure 4. Experiment scenario.

Due to a IEEE 802.15.4 standard limition, the enddevices only communicate with a PAN coordinator or a router device. Therefore the routing protocol AODV adopted in our topology can be simplified. This change was made in NS-2 routing kernel.

Within this context, an industrial monitoring application was used for the performance evaluation of temporal data compression Swinging Door algorithm. Basically, 10,000 samples were extracted from a real database system for gas injection monitoring in an onshore oil field [12] and then replicated for all end-device models. Therefore, up to 70,000 real samples can be transmitted in the simulated network scenario.

The development of the Swinging Door algorithm was made in the application layer of NS-2. The traffic pattern follows a constant bit rate with an interval configured by the user plus an uniform random range between 0 and 1 second. Scripts for automated configuration of patterns traffic have been developed to facilitate the interaction with the application.

The main feature of compression algorithm is the relation between the rate compression and the compression errors due to information losses. It is not interesting to have an high compression rate if the amount of distortion introduced by algorithm is too large. Therefore a tradeoff between both parameters must be determined. The analysis of this tradeoff in our experiments was evaluated through of mean square error for a linear interpolation mechanism on the compressed data transmitted around the network. The results described in the figure 5 do not suggest the use this algorithm for compression rate higher than $85 \%$.

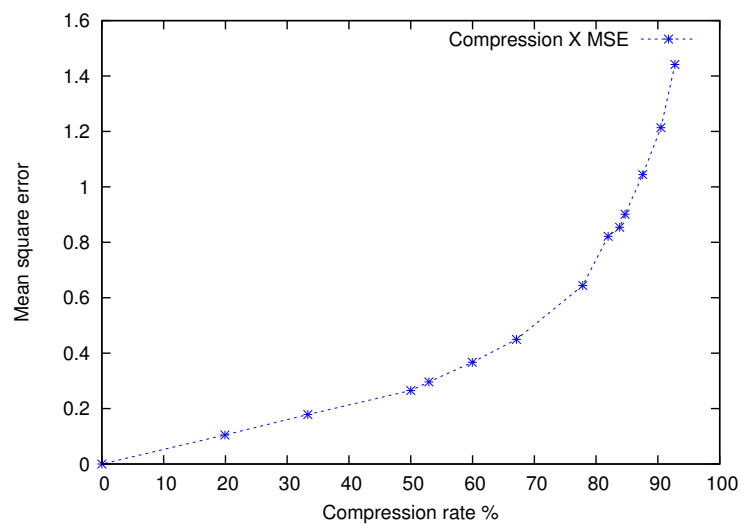

\section{Figure 5. Analysis of mean square error for the compression algorithm.}

It is clear that the use of a temporal data compression algorithm to eliminate the transmission of redundant information minimizes the energy consumption around the network. However is necessary to measure the influence of the compression rate on the energy consumption. Thus, in the figure 6 it is possible to see that the influence of the compression rate and the energy consumption do not have a linear relation, because the saved energy corresponds only to the transmission energy portion and is not related with the processing and reception activities. Thus, for example to $85 \%$ rate compression, the saved energy is around $65 \%$.

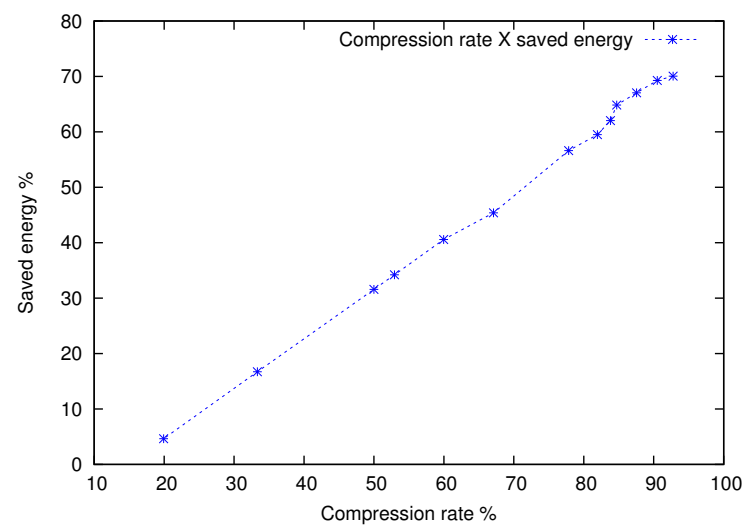

\section{Figure 6. Influence of compression rate in the saved energy.}

Saved energy is very important to extend the lifetime of batteries that supply energy to sensor nodes. In our experiments, the analyse of batteries' lifetime was made based 
on 3 parameters: simulation runtime (15,000 seconds), 2 AA batteries $(2300 \mathrm{mAh}, 1.5 \mathrm{~V})$ and the mean energy consumption around the network without the Swinging Door algorithm $(10.53 \mathrm{~mW})$. The equation 1 evaluates the lifetime through these parameters.

$$
\text { lifetime }(\text { days })=\frac{\text { runtime } \times \text { energy batteries }}{\text { energy used } \times 3600 \times 24}
$$

Figure 7 shows the relation between compression rate and the network lifetime. If the Swinging Door algorithm is not used, the network lifetime was assessed to be around 400 days. However, when this algorithm is used, the network lifetime is highly extended. For example, using a $85 \%$ compression rate, the network lifetime is extended to 1,200 days, i.e., a ratio of 3 when compared to the case where the Swinging Door algorithm is not used.

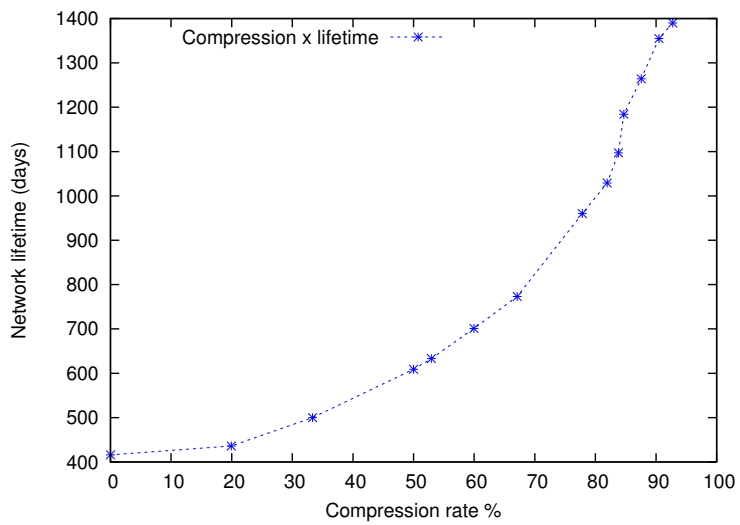

\section{Figure 7. Network lifetime to different com- pression rate.}

Another information used in our simulation was the network stability. This information describes the relation between the sent data packets and received data packtes.

Figure 8 shows that the adoption of the Swinging Door algorithm can maximize the network stability for different applications range. This improvement is due to the elimination of redundant data transmissions, reducing the collision likelihood as there are less messages being transmitted. On the other hand, when the Swinging Door algorithm is not used the network stability performance decreases.

Investigating on the NS-2 log, it was verified that some problems occur due to the hidden terminal problem. This network problem happens when nodes that are outside the range of other nodes send packets simultaneously to the sink node. In a situation like this, nodes cannot sense the carrier and the CSMA/CA does not work. As described in figure 8 , we can see that in a star topology with radius up to $25 \mathrm{~m}$, the hidden terminal problem occurs. In this situation, due to $50 \mathrm{~m}$ static sensibility on sensor nodes, those sensor nodes that are located on opposite side of topology do not sensing each other.

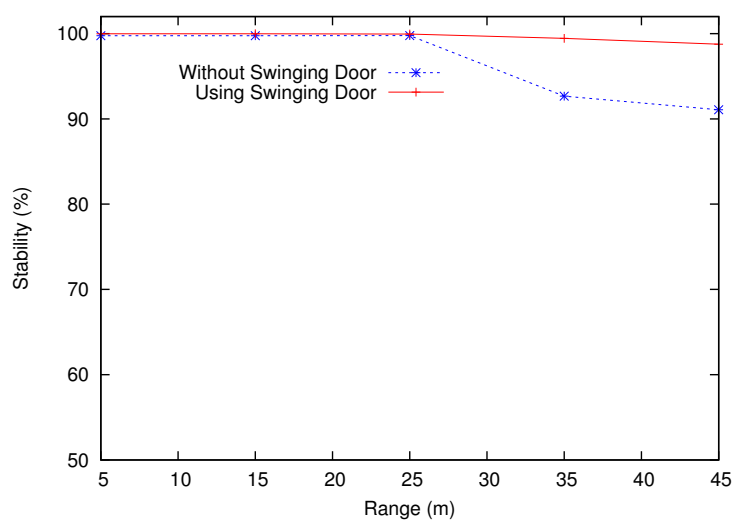

Figure 8. Percentage of successfull transmitted data packets.

\section{Conclusion}

In this paper, the performance of the temporal compression algorithm Swinging Door for Wireless Sensor Network in a monitoring application have been investigated. Simulations made using the NS-2 tool shown that the adoption of the Swinging Door algorithm can eliminate the redundant data transmission around network. Thus the energy consumption in the sensor nodes can be minimized.

The obtained results show that the relation between compression rate of algorithm and nodes consumption energy is not linear and for compression rates around $85 \%$, the network lifetime is tripled.

Analysis made in network stability shown that the algorithm can minimize the hidden terminal problem through the network load reduction.

A suggestion for further work is related with the adoption of a faulty tolerance mechanism in noisy channel where the algorithm can be analysed.

\section{References}

[1] I. S. 802.15.4. Wireless Medium Access Control (MAC) and Physical Layer (PHY) Specifications for Low-Rate Wireless Personal Area Networks (WPANs). IEEE Computer Society, Revision of IEEE Std 802.15.4-2003), 2006.

[2] I. F. Akyildiz, W. Su, Y. Sankarasubramaniam, and E. Cayirci. Wireless sensor networks: a survey. Computer Networks (Amsterdam, Netherlands: 1999), 38(4):393422, 2002.

[3] B. Bougard, F. Catthoor, D. C. Daly, A. Chandrakasan, and W. Dehaene. Energy efficiency of the ieee 802.15.4 standard in dense wireless microsensor networks: Modeling and improvement perspectives. In DATE '05: Proceedings of the conference on Design, Automation and Test in Europe, pages 196-201, Washington, DC, USA, 2005. IEEE Computer Society. 
[4] E. H. Bristol. Swinging door trending: Adaptive trend recording? ISA National Conf. Proc., pages 749-753, 1990.

[5] S. Bu and F. Naghdy. Service discovery in wireless ad-hoc control networks. In Intelligent Sensors, Sensor Networks and Information Processing Conference, pages 157- 162, Melbourne, Australia, 2005. IEEE.

[6] V. C. Gungor and F. C. Lambert. A survey on communication networks for electric system automation. Comput. Networks, 50(7):877-897, 2006.

[7] M. J. Jianliang Zheng. A Comprehensive Performance Study of IEEE 802.15.4. IEEE Press Book, 2004.

[8] I. Johnstone, J. Nicholson, B. Shehzad, and J. Slipp. Experiences from a wireless sensor network deployment in a petroleum environment. In IWCMC '07: Proceedings of the 2007 international conference on Wireless communications and mobile computing, pages 382-387, New York, NY, USA, 2007. ACM.

[9] T. Z. Kazem Sohraby, Daniel Minoli. Wireless sensor networks: technology, protocols, and applications. 978-0471-74300-2. John Wiley, 2007.

[10] K. Klues, J. Hoffert, and O. Orjih. Configuring the ieee 802.15.4 mac layer for single-sink wireless sensor networks. Technical report, Washington University in St. St. Louis, 2005.

[11] L. Krishnamurthy, R. Adler, P. Buonadonna, J. Chhabra, M. Flanigan, N. Kushalnagar, L. Nachman, and M. Yarvis. Design and deployment of industrial sensor networks: experiences from a semiconductor plant and the north sea. In SenSys '05: Proceedings of the 3rd international conference on Embedded networked sensor systems, pages 6475, New York, NY, USA, 2005. ACM.

[12] E. Leao, L. Guedes, and F. Vasques. Implementation of an event-triggered smart sensor network architecture based on the ieee 802.15.4 standard. Fieldbuses and Networks in Industrial and Embedded Systems, 2007 7th IFAC International Conference on, pages 179-184, 7-9 November 2007.

[13] A. Willig, K. Matheus, and A. Wolisz. Wireless technology in industrial networks. Proceedings of the IEEE, 93(6):1130-1151, June 2005. 